\title{
Noisy regression and classification with continuous multilayer networks
}

\author{
M. Ahr ${ }^{1}$, M. Biehl ${ }^{1}$, and R. Urbanczik ${ }^{2}$ \\ ${ }^{1}$ Institut für Theoretische Physik \\ Julius-Maximilians-Universität Würzburg \\ Am Hubland, D - 97074 Würzburg, Germany \\ ${ }^{2}$ Neural Computing Research Group \\ Aston University \\ Aston Triangle, Birmingham B4 7ET, UK
}

November 14, 2018

\begin{abstract}
We investigate zero temperature Gibbs learning for two classes of unrealizable rules which play an important rôle in practical applications of multilayer neural networks with differentiable activation functions: classification problems and noisy regression problems. Considering one step of replica symmetry breaking, we surprisingly find that for sufficiently large training sets the stable state is replica symmetric even though the target rule is unrealizable. Further the classification problem is shown to be formally equivalent to the noisy regression problem.
\end{abstract}

Neural networks with differentiable activation functions play an important rôle in practical applications [1]. Besides being used for regression, they are often applied to classification problems as well, since gradient based methods are available for training such networks. In both cases, given a training set of $P$ input/output pairs $\left(\vec{\xi}_{\mu}, \theta_{\mu}\right)$, $\vec{\xi}_{\mu} \in \mathbb{R}^{N}, \theta_{\mu} \in \mathbb{R}$, one adapts the network with output $\sigma$ to minimize a cost function which measures the deviation between $\sigma\left(\vec{\xi}_{\mu}\right)$ and the target output $\theta_{\mu}$.

For the regression problem we shall assume that the target output is a function $\tau$ of the input, corrupted by additive noise, so $\theta_{\mu}:=\tau\left(\vec{\xi}_{\mu}\right)+\gamma \nu_{\mu}$. The noise terms $\nu_{\mu}$ are independent and normally distributed. An appropriate cost function then is the quadratic error

$$
H=P \epsilon_{t}=\frac{1}{2} \sum_{\mu=1}^{P}\left(\sigma\left(\vec{\xi}_{\mu}\right)-\theta_{\mu}\right)^{2}
$$


We call $\epsilon_{t}$, the mean energy per example, training error. The main goal of learning, however, is to minimize the prediction error $\epsilon_{p}$, defined as the expectation value of the training error on a new example, that is $\epsilon_{p}=\left\langle(\sigma(\vec{\xi})-\theta(\vec{\xi}))^{2}\right\rangle / 2$, where the average is performed over the distribution of inputs and the randomness of $\theta$ in the presence of noise.

In classification problems only a binary label is available for the examples and we shall assume that $\theta_{\mu}=\lambda \operatorname{sign}\left(\tau\left(\vec{\xi}_{\mu}\right)\right)$. Here $\tau$ is some function of the input and $\lambda$ is a tunable parameter. One is then mainly interested in the sign of the networks output, that is the goal of learning is to minimize the classification error

$$
\epsilon_{c}=\langle\Theta(-\sigma(\vec{\xi}) \tau(\vec{\xi}))\rangle
$$

where $\Theta$ is the Heavyside step function. However the empirical mean of this performance measure

$$
P^{-1} \sum_{\mu=1}^{P} \Theta\left(-\sigma\left(\vec{\xi}_{\mu}\right) \theta_{\mu}\right)
$$

is piecewise constant and cannot be optimized using e.g. backpropagation. While the sample complexity of training multilayer networks based on (3) has been analysed in [2, 3, \#], practical applications of neural networks [11, 5, 6] typically use the differentiable cost function (11) even for classification tasks. So for the purposes of training, classification is mapped onto regression, and the question arises how this affects the generalization behaviour. (Alternative cost functions have been studied in the context of online learning [7].)

Here we present a theoretical investigation of the two learning problems. We focus on a simple two-layered student network which consists of $K$ hidden units with activation function $\mathrm{g}(x)=\operatorname{erf}(x / \sqrt{2})$ and $N$-dimensional weight vectors $\left\{\vec{J}_{i}\right\}_{i=1}^{K}$, where $\vec{J}_{i}^{2}=N$. The output unit is linear and has weights fixed to the value $1 / \sqrt{K}$. Then, the output of this network which is called "soft-committee machine" [8, 9] is

$$
\sigma(\vec{\xi})=\frac{1}{\sqrt{K}} \sum_{i=1}^{K} \mathrm{~g}\left(\frac{\overrightarrow{J_{i}} \cdot \vec{\xi}}{\sqrt{N}}\right) .
$$

The target function $\tau(\vec{\xi})$ will be given by a soft-committee machine with the same number of hidden units as the student network and weight vectors $\left\{\vec{B}_{i}\right\}_{i=1}^{K}$, where $\vec{B}_{i} \cdot \vec{B}_{j}=N \delta_{i j}$. So the classification problem is perfectly learnable in the sense that the student network can achieve $\epsilon_{c}=0$ if its weight vectors become identical to those of the teacher network. Further, we assume the components of the examples to be independent random numbers with mean zero and unit variance.

We use the well-known replica formalism to investigate these problems in the thermodynamic limit $N \rightarrow \infty$. This requires the calculation of the quenched free energy

$$
F=-\frac{1}{\beta}\langle\ln Z\rangle=-\left.\frac{1}{\beta} \frac{\partial}{\partial n} \ln \left\langle Z^{n}\right\rangle\right|_{n=0}
$$


where $Z^{n}$ is the partition function $\int d \mu\left(\left\{\overrightarrow{J_{i}^{a}}\right\}\right) \exp \left(-\beta \sum_{a=1}^{n} H\left(\left\{{\overrightarrow{J_{i}^{a}}}_{i=1}^{K}\right)\right)\right.$ of $n$ replicas (labeled $a, b=1,2,3, \ldots)$ of the student network [11, 12]. Here $H$ is interpreted as the energy of a system which is in thermal equilibrium at a temperature $T=1 / \beta$. In the limit of zero temperature, $\beta \rightarrow \infty, F$ is the optimal value of the energy which can be achieved by minimizing $H$ with respect to the network weights.

Introducing an additional integration over the order parameters $Q_{i j}^{a b}:=\vec{J}_{i}^{a} \cdot \vec{J}_{j}^{b} / N$ and $R_{i j}^{a}:=\vec{J}_{i}^{a} \cdot \vec{B}_{j} / N$, which is performed as a saddle point integration in the limit of large $N$, we find for moments of the partition function, $\ln \left\langle Z^{n}\right\rangle=-\left.N\left(\alpha K G_{r}+s\right)\right|_{\text {extr- }}$. Here $G_{r}$ is an effective Hamiltonian and the entropy term $s=(1 / 2) \ln \operatorname{det} \underline{\underline{C} \text {, where }}$ $\underline{\underline{C}}$ is the $K(n+1) \times K(n+1)$-dimensional matrix of the order parameters [10]. We have further introduced the rescaled number of examples, $\alpha=P /(N K)$.

In the following we restrict ourselves to the limit of large $K$. We make a sitesymmetric Ansatz for the dependence of the order parameters on the site indices $i, j$ :

$$
\begin{aligned}
R_{i j}^{a} & =\delta_{i j}\left(\frac{\hat{R}^{a}}{K}+\Delta^{a}\right)+\left(1-\delta_{i j}\right) \frac{\hat{R}^{a}}{K} \\
Q_{i j}^{a b} & =\delta_{i j}\left(\frac{\hat{Q}^{a b}}{K}+\delta^{a b}\right)+\left(1-\delta_{i j}\right) \frac{\hat{Q}^{a b}}{K}
\end{aligned}
$$

The scaling of the unspecialized order parameters with the number of hidden units results from the condition that the outputs of the students $\sigma^{a}$ must be of order 1 in the limit of infinite $K$. In this limit the calculation of $G_{r}$ can be carried out analytically since the joint distribution of the $\sigma^{a}$ and $\tau$ becomes Gaussian [ [ [].

For the regression problem, using a one step replica symmetry breaking Ansatz, we obtain in the limit $n \rightarrow 0$ :

$$
G_{r}^{0}=\frac{1}{2}\left(\frac{X_{1}}{X_{2}}+\frac{m-1}{m} \ln X_{3}+\frac{1}{m} \ln X_{2}\right) ;
$$

where

$$
\begin{aligned}
& X_{1}=\beta\left(v^{0}-2 w+1 / 3+\gamma^{2}\right) \\
& X_{2}=1+\beta\left(u+(m-1) v^{1}-m v^{0}\right) \\
& X_{3}=1+\beta\left(u-v^{1}\right)
\end{aligned}
$$

$u=1 / 3+\hat{Q} / \pi, v^{1}=f\left(\delta^{1}, \hat{Q}^{1}\right), v^{0}=f\left(\delta^{0}, \hat{Q}^{0}\right)$, and $w=f(\Delta, \hat{R})$ are the covariances of the $\sigma^{a}$ and $\tau$, where

$$
f(x, y)=\frac{2}{\pi} \arcsin \left(\frac{x}{2}\right)+\frac{y}{\pi} .
$$

It is easy to calculate $s$ and to perform the limit $n \rightarrow 0$ to obtain the entropy term $s^{0}$ in the free energy which is the same as for hard committee machines [ order parameter $\Delta$ indicates specialization of the network: if $\Delta=0$, the network configuration is unspecialized, i.e. a weight vector of the student network has the same overlap $(\hat{R} / K)$ with all weight vectors of the teacher network, whereas a positive $\Delta$ 
indicates a specialized configuration where each of the student vectors has a greater overlap $(\hat{R} / K+\Delta)$ with one of the teacher vectors than with the others. $\hat{Q} / K$ is the cross-overlap between different weight vectors of a student. The remaining order parameters $\hat{Q}^{0}, \hat{Q}^{1}, \delta^{0}, \delta^{1}$ and $m$ parametrize the distribution of overlaps between the weight vectors of different students. Note that as in [10], using the saddle point equations for the free energy, one may analytically eliminate the unspecialized order parameters $\hat{R}, \hat{Q}, \hat{Q}^{0}$ and $\hat{Q}^{1}$.

In terms of the order parameters the prediction error for the regression problem is given by:

$$
\epsilon_{p}=\frac{1}{3}+\frac{\hat{Q}}{2 \pi}-\frac{\hat{R}}{\pi}-\frac{2}{\pi} \arcsin \left(\frac{\Delta}{2}\right)+\frac{\gamma^{2}}{2} .
$$

The replica calculation for the classification problem is analogous. It yields the same entropy $s^{0}$ and a $G_{r}^{0}$ of the form (8) with identical $X_{2}$ and $X_{3}$ but

$$
X_{1}=\beta\left(v^{0}-2 w \lambda \sqrt{6 / \pi}+\lambda^{2}\right) .
$$

For the prediction and classification error one finds

$$
\begin{aligned}
\epsilon_{p} & =\frac{1}{6}+\frac{\hat{Q}}{2 \pi}-\lambda \sqrt{\frac{6}{\pi}}\left(\frac{2}{\pi} \arcsin \left(\frac{\Delta}{2}\right)+\frac{\hat{R}}{\pi}\right)+\frac{\lambda^{2}}{2} \\
\epsilon_{c} & =\frac{1}{\pi} \arccos \left[\left(\frac{2}{\pi} \arcsin \left(\frac{\Delta}{2}\right)+\frac{\hat{R}}{\pi}\right)\left(\frac{1}{9}+\frac{\hat{Q}}{3 \pi}\right)^{-\frac{1}{2}}\right] .
\end{aligned}
$$

In the limit of large sample size $P$ the training error $\epsilon_{t}$ will converge to $\epsilon_{p}$. So for the classification error to become zero the value of $\lambda$ must be chosen so that the minima of $\epsilon_{p}$ and $\epsilon_{c}$ coincide. Note that the order parameters are constrained by the fact that the vectors $\vec{a}:=(1 / N) \sum_{i=1}^{K} \vec{J}_{i}$ and $\vec{b}:=\vec{B}_{j}$ must fulfill $(\vec{a} \cdot \vec{b})^{2} \leq \vec{a}^{2} \vec{b}^{2}$, which demands $\hat{Q} \geq(\Delta+\hat{R})^{2}-1$. Minimizing the prediction error (11) under this restriction, we obtain $\Delta=1$ and $\hat{Q}=\hat{R}=0$ (student and teacher network identical, $\epsilon_{c}=0$ ) only for $\lambda=\lambda_{o}=\sqrt{\pi / 6}$. This is the optimal value of $\lambda$ which allows asymptotically perfect classification. Inserting $\lambda=\lambda_{o}$ in equation (10) and comparing to (8), one finds that in this case the free energy of the classification problem is identical to that of a noisy regression problem with $\gamma=\gamma_{0}=\sqrt{\pi / 6-1 / 3}$. In the sequel we shall only consider the case $\lambda=\lambda_{0}$ for the classification problem.

We focus on the limit of zero temperature and the construction of this limit depends on whether a zero training error is achievable. Denoting this critical capacity by $\alpha_{\mathrm{c}}(\gamma)$, we find that $\alpha_{\mathrm{c}}(\gamma)$ decreases to 0 with increasing $\gamma$ and $\alpha_{\mathrm{c}}(\gamma) \rightarrow 1$ as $\gamma \rightarrow 0$. This is explained by the fact that the noise increases the magnitude of the target outputs. This correlates the hidden units of the student and thus reduces the storage capacity.

Below $\alpha_{\mathrm{c}}(\gamma)$ we find an unspecialized replica symmetric solution with $\Delta=\delta^{1}=$ $\delta^{0}=0$. Above $\alpha_{\mathrm{c}}(\gamma)$ one finds $\delta^{1} \rightarrow 1$ for $\beta \rightarrow \infty$ and the appropriate scaling is 
$1-\delta^{1}=\hat{\eta} / \beta$ where $\hat{\eta}$ is $\mathcal{O}(1)$. To achieve nontrivial results $m$ must also be scaled with $\beta$ and we reparametrize $m=\hat{m} / \beta$. Then for $\alpha>\alpha_{\mathrm{c}}(\gamma)$ the zero temperature free energy functional is given by:

$$
\begin{aligned}
\frac{2 F}{N K} & =\alpha\left\{\frac{1-2 z(\Delta)+z\left(\delta^{0}\right)+3 \gamma^{2} \pi /(\pi-3)}{\kappa \hat{\eta}+\hat{m}\left(1-z\left(\delta^{0}\right)\right)+3 \pi /(\pi-3)}\right. \\
& \left.+\frac{1}{\hat{m}} \ln \left[\frac{\kappa \hat{\eta}+\hat{m}\left(1-z\left(\delta^{0}\right)\right)+3 \pi /(\pi-3)}{\kappa \hat{\eta}+3 \pi /(\pi-3)}\right]\right\} \\
& -\frac{\delta^{0}-(\Delta)^{2}}{\hat{\eta}+\hat{m}\left(1-\delta^{0}\right)}-\frac{1}{\hat{m}} \ln \left[\frac{\hat{\eta}+\hat{m}\left(1-\delta^{0}\right)}{\hat{\eta}}\right]
\end{aligned}
$$

where $\kappa=(2 \sqrt{3}-3) /(\pi-3)$ and $z(x)=(-3 / \pi)(x-2 \arcsin (x / 2))$. The replica symmetric case may be recovered by either taking the limit $\hat{m} \rightarrow 0$ or the limit $\delta^{0} \rightarrow 1$.

These equations still admit an at least metastable unspecialized solution with $\Delta=$ 0 for all $\alpha>\alpha_{\mathrm{c}}(\gamma)$. But now replica symmetry is broken in this solution, and this also holds in the noiseless case $\gamma=0$. Above a second critical $\alpha$ the stable solution is specialized $(\Delta>0)$ and remarkably even in the noisy case this specialized solution does not exhibit replica symmetry breaking.

The lifting of RSB with the onset of specialization is illustrated in Figure 1 for $\gamma=\gamma_{0}$. Fixing $\Delta$ and maximizing (13) w.r.t. to the remaining order parameters corresponds to calculating the free energy of a system with a state space constrained to vectors yielding a specialized student/teacher overlap of $\Delta$. At the maximum $F / P$ is the training error of the constrained system shown in Figure 1.

The physically relevant states in the case of training with unconstrained $\Delta$ are given by the minima of these curves. Both in the RS and the RSB parametrizations we find a local minimum at $\Delta=0$, which corresponds to a metastable unspecialized configuration of the system. Here the RSB solution yields a greater free energy than the RS solution and therefore is the only physically relevant solution.

With increasing $\Delta$ both curves approach each other, and the RSB and RS solutions merge at $\Delta \approx 0.78$, i.e. for sufficiently large $\Delta$ there is no replica symmetry breaking.

There is a second minimum of the free energy at $\Delta \approx 0.87$ which corresponds to a replica symmetrical specialized phase of the learning with unconstrained $\Delta$ which yields a lower free energy than the unspecialized solution and therefore is the globally stable configuration.

In general, we find the following scenario which is illustrated in the right panel of Figure 1 for $\gamma=\gamma_{0}$. For all values of $\alpha$ there is an unspecialized solution with constant prediction error $\left(\epsilon_{p}=1 / 3-1 / \pi+\gamma^{2} / 2\right)$. Replica symmetry is broken in this solution for $\alpha>\alpha_{\mathrm{c}}(\gamma)$. Beyond a second critical $\alpha$ the unspecialized solution is only metastable and the stable solution is specialized and replica symmetric. In the noiseless case, the two critical values of $\alpha$ coincide, and thus replica symmetry is never broken in the stable state. In the noisy case, the prediction error decays as $1 / \alpha$ to its asymptotical value $\gamma^{2} / 2$ in the specialized phase. 

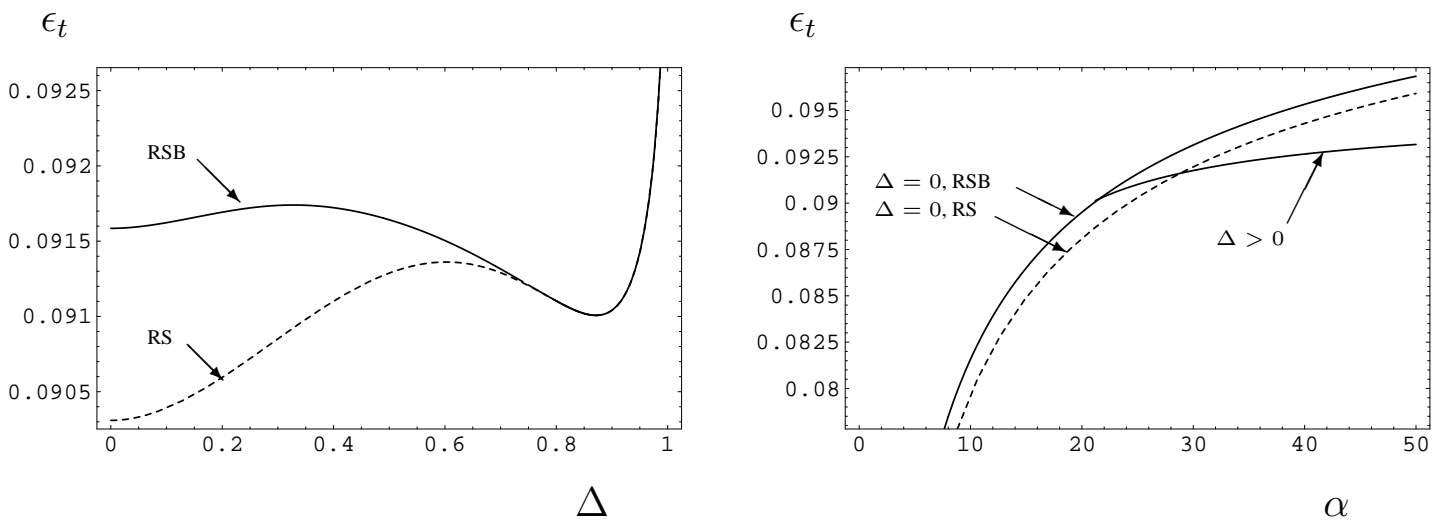

Figure 1: Results for the classification problem and for the noisy regression problem with $\gamma=\gamma_{0}$. Left panel: $\epsilon_{t}(\Delta)$ for $\alpha=25$. Dashed line: replica symmetrical solution, solid line: one-step replica symmetry breaking solution. Right panel: $\epsilon_{t}(\alpha)$. At $\alpha_{\mathrm{c}}\left(\gamma_{0}\right) \approx 0.3$ replica symmetry is broken. At $\alpha \approx 21.5$ replica symmetry is restored with the onset of specialization. The dashed line shows the (wrong) results of a replica symmetrical calculation.

For the classification problem, $\gamma=\gamma_{0}$, the $1 / \alpha$ decay of the prediction error translates into the following asymptotics of the classification error:

$$
\epsilon_{c} \sim \frac{\sqrt{\frac{\pi}{2}-1}}{\pi^{\frac{1}{4}}} \frac{1}{\sqrt{\alpha}} .
$$

This slow decay of $\epsilon_{c}$ reflects the cost of treating the classification problem as a regression problem and thus mapping a realizable case onto an unrealizable one. Based on the results of [2] one would expect a $1 / \alpha$ asymptotics of the classification error, if the hard cost function (3) would be used instead of the quadratic deviation (11). Thus future research into batch learning should investigate the use of cost functions like the ones proposed in [7] for the online scenario.

For the general case of noisy regression, it is remarkable that replica symmetry breaking is only a transient phenomenon in that the specialized state which is the stable one for large $\alpha$ is replica symmetric even in this unrealizable scenario.

Part of this work was carried out during the Seminar on Statistical Physics of Neural Networks at the Max-Planck-Institut für Physik komplexer Systeme in Dresden. Further this work was supported by a British Council grant (British-German Academic Research Collaboration, programme project 1037) and a DAAD grant (project number 9818105).

We thank Georg Reents and Enno Schlösser for stimulating discussions and a critical reading of the manuscript. 


\section{References}

[1] J. Hertz, A. Krogh, R.G. Palmer. Introduction to the theory of neural computation. Addison Wesley, Redwood City 1991

[2] H. Schwarze, J. Hertz. Europhys. Lett. 21, 785, 1993 and H. Schwarze. J. Phys. A 26, 5781, 1993

[3] H. Schwarze, J. Hertz. in Advances in Neural Information Processing Systems V. Hrg. S.J. Hanson et. al. , Morgan Kaufmann, San Mateo, 1993

[4] R. Urbanczik. J. Phys. A 28, 7097, 1995 and R. Urbanczik. Phys. Rev. E 58 (2), 2298, 1998

[5] C. Bishop, Neural Networks for Pattern Recognition. Oxford University Press, Oxford, 1995

[6] E. Eisenstein, I. Kanter. Europhys. Lett. 21(4), 501, 1993

[7] N. Barkai, H.S. Seung, H. Sompolinsky. Phys. Rev. Lett. 75, 1415, 1995

[8] D. Saad, S.A. Solla. Phys. Rev. Lett 74, 4337, 1995 and D. Saad, S. A. Solla. Phys. Rev. E 52, 4225, 1995

[9] K. Kang, J.-H. Oh, C. Kwon, Y. Park. Phys. Rev. E 48, 4805, 1993

[10] M. Ahr, M. Biehl, R. Urbanczik. to be published in European Physical Journal B

[11] H.S. Seung, H. Sompolinsky, N. Tishby. Phys. Rev. A 45, 6056, 1992

[12] T.L.H. Watkin, A. Rau, M. Biehl. Rev. Mod. Phys. 65 (2), 499, 1993 